\title{
A broadband achromatic metalens for focusing and imaging in the visible
}

\section{Citation}

Chen, Wei Ting, Alexander Y. Zhu, Vyshakh Sanjeev, Mohammadreza Khorasaninejad, Zhujun Shi, Eric Lee, and Federico Capasso. 2018. "A Broadband Achromatic Metalens for Focusing and Imaging in the Visible." Nature Nanotechnology 13 (3) (January 1): 220-226. doi:10.1038/ s41565-017-0034-6.

\section{Published Version}

doi:10.1038/s41565-017-0034-6

\section{Permanent link}

http://nrs.harvard.edu/urn-3:HUL.InstRepos:35058850

\section{Terms of Use}

This article was downloaded from Harvard University's DASH repository, and is made available under the terms and conditions applicable to Other Posted Material, as set forth at http:// nrs.harvard.edu/urn-3:HUL.InstRepos:dash.current.terms-of-use\#LAA

\section{Share Your Story}

The Harvard community has made this article openly available.

Please share how this access benefits you. Submit a story.

\section{Accessibility}




\title{
A broadband achromatic metalens for focusing and imaging in the visible
}

\author{
Wei Ting Chen ${ }^{1}$, Alexander Y. Zhu ${ }^{1}$, Vyshakh Sanjeev ${ }^{1,3}$, Mohammadreza \\ Khorasaninejad ${ }^{1}$, Zhujun Shi $^{2}$, Eric Lee ${ }^{1,3}$ and Federico Capasso ${ }^{1, *}$ \\ ${ }^{I}$ Harvard John A. Paulson School of Engineering and Applied Sciences, Harvard University, \\ Cambridge, Massachusetts 02138, USA \\ ${ }^{2}$ Department of Physics, Harvard University, Cambridge, Massachusetts 02138, USA \\ ${ }^{3}$ University of Waterloo, Waterloo, ON N2L 3G1, Canada \\ *Corresponding author: capasso@seas.harvard.edu
}

A key goal of metalens research is to achieve wavefront shaping of light using optical elements with thicknesses on the order of the wavelength. Such miniaturization is expected to lead to compact, nanoscale optical devices with applications in cameras, lighting, displays and wearable optics. However, retaining functionality while reducing device size has proven particularly challenging. For example, so far there has been no demonstration of broadband achromatic metalenses covering the entire visible spectrum. Here we show that by judicious design of nanofins on a surface, it is possible to simultaneously control the phase, group delay and group delay dispersion of light, thereby achieving a transmissive achromatic metalens with large bandwidth. We demonstrate diffraction-limited achromatic focusing and achromatic imaging from 470 to $670 \mathrm{~nm}$. Our metalens is comprised of only a single layer of nanostructures whose thickness is on the order of the wavelength, and does not involve spatial multiplexing 
or cascading. While this initial design (numerical aperture of 0.2 ) has an efficiency of about $20 \%$ at $500 \mathrm{~nm}$, we discuss ways in which our approach may be further optimized to meet the demand of future applications. 
Conventional refractive optical components are generally bulky, costly, and timeconsuming to manufacture with high precision ${ }^{1}$. These are significant limitations, particularly for applications such as portable and wearable devices. In recent years, metasurfaces have emerged as a versatile platform for wavefront shaping. Since the phase is accurately controlled by subwavelength-spaced structures with thicknesses at the wavelength scale or below, many compact optical devices based on metasurfaces have been demonstrated. These include flat lenses $^{2-4}$, polarimeters $^{5-7}$, axicons ${ }^{8,9}$, polarization elements ${ }^{10-12}$ and holograms ${ }^{13-15}$. However, these devices are highly chromatic despite being comprised of weakly dispersive materials. This can be attributed to two separate factors: dispersion arising from a periodic lattice (see Fig. S1 for detailed discussion), as well as light confinement in either a resonant or guided manner. Previous works have addressed this challenge by using multiple coupled resonances to tailor phase profiles at several discrete frequencies ${ }^{16,17}$, stacking/stitching several layers of meta-surfaces ${ }^{18}$, increasing the phase modulation to be more than $2 \pi$ radians (so-called "multi-order diffractive lenses") ${ }^{19}$ or engineering the dispersion ${ }^{20-}$ ${ }^{23}$. Recently, achromatic focusing at green wavelengths (with a $60 \mathrm{~nm}$ bandwidth) in a reflective metalens was achieved ${ }^{24}$. Other groups have experimentally demonstrated achromatic metalenses in the near-infrared with bandwidths of a few tens of terahertz $(\mathrm{THz})^{25-27}$. However, none of these works demonstrated achromatic imaging. 
Here, we demonstrate the ability to engineer the frequency dependent phase profile $\varphi(\vec{r}, \omega)$, and thereby achieve arbitrary control of metalens dispersion over a large continuous bandwidth in the visible. This is made possible by separately engineering the group delay and group delay dispersion of each constituent nanostructure, independent of its phase, at a given frequency. This is distinct from other approaches, particularly that of Wang et. al. ${ }^{27}$. They utilized plasmonic resonances without considering group delay dispersion, and therefore lacked a systematic, general way to implement metalenses of different dispersion. As a proof of concept, we demonstrate metalenses with tailored dispersion, including achromatic metalenses with diffractionlimited focusing covering nearly the entire visible (from $470 \mathrm{~nm}$ to $670 \mathrm{~nm}$ ). The achromatic metalens is also capable of performing white light imaging. Finally, we design and model a metasurface which, when patterned over a commercial spherical lens, renders it achromatic and diffraction-limited across the visible spectrum.

\section{Principle of achromatic metalenses}

As an initial example, consider the achromatic metalens shown in Fig. 1(a). The relative phase provided by the metalens elements with respect to the center follows ${ }^{3}$ :

$$
\varphi(r, \omega)=-\frac{\omega}{c}\left(\sqrt{r^{2}+F^{2}}-F\right)
$$

where $\omega, c, r$ and $F$ are angular frequency, light speed, radial coordinate, and focal length, respectively. This spatial- and frequency-dependent phase profile $\varphi(r, \omega)$ 
implies that at a given $r$, the metalens provides different transverse wavevectors $k_{r}=\frac{\partial \varphi(r, \omega)}{\partial r}$ so that different wavelengths are deflected by the same angle. Equation 1 can be Taylor-expanded near a design frequency $\omega_{d}$ as

$$
\varphi(r, \omega)=\varphi\left(r, \omega_{d}\right)+\left.\frac{\partial \varphi(r, \omega)}{\partial \omega}\right|_{\omega=\omega_{d}}\left(\omega-\omega_{d}\right)+\left.\frac{\partial^{2} \varphi(r, \omega)}{\partial \omega^{2}}\right|_{\omega=\omega_{d}}\left(\omega-\omega_{d}\right)^{2}+\ldots
$$

Equation 2 indicates that to achieve achromatic focusing within a given bandwidth $\Delta \omega$ around $\omega_{d}$, an optical element placed at a radial coordinate $r$ needs to satisfy not only the required relative phase $\left(\varphi\left(r, \omega_{d}\right)\right)$, but also the higher-order derivative terms, which determine the metalens dispersion. $\frac{\partial \varphi(r, \omega)}{\partial \omega}$ and $\frac{\partial^{2} \varphi(r, \omega)}{\partial \omega^{2}}$ are the relative group delay and group delay dispersion, and are typically of the order of femto-second (fs) and femto-second square $\left(\mathrm{fs}^{2}\right)$ in the visible. Conventional diffractive lenses only satisfy the required phase, i.e. the phase profile at a design frequency. The neglect of these derivative terms results in chromatic effects. An intuitive interpretation of each term of Eq. 2 is shown in Fig. 1(a). The first term leads to a spherical wavefront (yellow line in Fig. 1(a)). The group delay term compensates for the difference in the wavepackets' arrival times at the focus, while the high-order derivative terms (group delay dispersion etc.) ensure that the outgoing wavepackets are identical. The net effect is the minimization of the spread in the arrival times of wavepackets at the focus to ensure they constructively interfere. The smaller the time spread, the larger the bandwidth achievable. Therefore, in order to realize diffraction-limited focusing for a broad 
bandwidth, both phase and group delay, as well as higher order terms, need to be considered.

To account for the dispersion of a metalens, the focal length $F$ in Eq. 1 can be parametrized as:

$$
F=k \times \omega^{n}
$$

where $k$ is a positive constant and $n$ is a real number. The dispersion of a metalens can thus be designed to arbitrary specification by substituting different values for $n$ (a real number). We refer to the metalenses with $n=0$ and $n=1$ as achromatic and diffractive metalenses hereafter. The diffractive metalens possesses a focal length shift similar to Fresnel lenses. From Eq. 3, the positive/negative values of $n$ imply that shorter/longer wavelengths are focused farther from/closer to the metalens, respectively. The larger the absolute value of $n$, the farther the separation between the focal spots of two wavelengths, resulting in stronger dispersion. Figure 1(b) and 1(c) show the required relative group delays and group delay dispersions as a function of radial coordinate for metalenses with NA $=0.2$ at $\lambda=530 \mathrm{~nm}$. For $n=2$ and -1 , they require non-negligible group delay dispersion to precisely control the focal length shift and achieve diffraction-limited focusing. Note that the required group delay and group delay dispersions are relatively small for $n=1$. This agrees with our previous observation that 
a diffractive metalens $(\mathrm{NA}=0.8)$ implemented using geometric phase can still focus light with a focal spot size approximately equal to a wavelength ${ }^{3}$.

\section{Independent control of phase and group delay}

To increase the degrees of freedom in our design, we utilized coupled phase-shift elements: two nanofins in close proximity, acting as coupled waveguides. Their geometric parameters are defined in Fig. 2(a), and scanning electron microscopic images from a fabricated metalens are provided in Fig. 2(b) and Fig. S2. It has been previously shown that coupled waveguides can support tunable dispersion, e.g. near zero group delay dispersions for a wide bandwidth ${ }^{28,29}$. For simplicity and without loss of generality, we first consider the optical properties of a single $\mathrm{TiO}_{2}$ nanofin, which can be fabricated using electron beam lithography followed by atomic layer deposition $^{30}$. When a left-handed circularly polarized beam passes through the nanofin, the transmitted light can be described by the Jones vector ${ }^{31}$ :

$$
\frac{t_{L}+t_{S}}{2}\left[\begin{array}{l}
1 \\
i
\end{array}\right]+\frac{t_{L}-t_{S}}{2} \cdot \exp (i 2 \alpha)\left[\begin{array}{c}
1 \\
-i
\end{array}\right]
$$

where $t_{L}$ and $t_{S}$ represent complex transmission coefficients when the incident light is polarized along the long and short axis of the nanofin, and $\alpha$ is the rotation angle. The second term in Eq. 4 is cross-polarized; we refer to its normalized amplitude squared as the polarization conversion efficiency hereafter. The phase shift is determined by the product $\left(t_{L}-t_{S}\right) \cdot \exp (i 2 \alpha)$, where $2 \alpha$ is a frequency-independent 
geometric phase equal to twice the rotation angle. This allows us to decouple the target phase profile from the required group delay and group delay dispersion (controlled by $\left.t_{L}-t_{S}\right)$. Figure 2(c) shows phase spectra for a nanofin with different rotation angles. The slope is approximately linear within a given bandwidth, and is independent of the rotation angle of the nanofin. This property allows us to design achromatic metalenses with a large bandwidth.

To gain physical insight into the dispersion design, each $\mathrm{TiO}_{2}$ nanofins can be regarded as truncated waveguide. Neglecting end reflections, the phase of the transmitted light after passing through the structure at a given coordinate $r$ is $\phi(r, \omega)=\frac{\omega}{c} n_{\text {eff }} h$, where $n_{\text {eff }}$ and $h$ represent the effective index and the height of the nanofin, respectively. The derivative with respect to angular frequency

$$
\frac{\partial \phi(r, \omega)}{\partial \omega}=\frac{h}{c} n_{e f f}+\frac{h \cdot \omega}{c} \frac{\partial n_{e f f}}{\partial \omega}
$$

yields the group delay: this is the ratio of the nanofin height to group velocity, which can be controlled by the nanofin dimensions and/or material used. Figure 2(d) shows a comparison of polarization conversion efficiency using the eigenmode solver and finite-difference time-domain (FDTD) methods (Methods). The good agreement verifies the validity of treating the nanofins as short waveguides. At higher frequencies the observed deviations result from the excitation of higher order modes and resonances within the nanofins ${ }^{32,33}$. Figure 2(e) shows the phases and polarization conversion 
efficiencies of five different nanofin elements. Their group delays were obtained using linear fitting of the phase spectra within a bandwidth of $120 \mathrm{~nm}$, centered at $530 \mathrm{~nm}$ (see Methods for details). This ensures that the group delay of an element fulfills the requirement shown in Fig. 1(b) and its GDD is close to zero for at least the $120 \mathrm{~nm}$ bandwidth being considered. However, as seen later in the text, our simulations and experimental results show that the metalens focal length is only weakly dependent on wavelength beyond this bandwidth, up to $670 \mathrm{~nm}$. Note that for low NA, the required range of group delay is proportional to the product of lens radius and NA (see Methods).

We designed and implemented metalenses with nanofin dimensions corresponding to a group delay range of about 5 femto-seconds; see Fig. S3 for a plot of polarization conversion efficiencies versus group delays.

\section{Achromatic focusing and imaging}

To demonstrate the versatility of our approach, we designed and fabricated an achromatic metalens $(n=0)$ as well as two other metalenses with $n=1$ and 2 . They all possess NA $=0.2$ at wavelength $\lambda=530 \mathrm{~nm}$. For $n=1$, i.e. a regular diffractive metalens, the phase profile was imparted by identical nanofins using the geometric phase ${ }^{34}$. The achromatic metalenses were designed by digitizing the required phase and group delay, which were then implemented by selecting elements from a library of various nanofin parameters. For $n=2$, we also selected elements with group delay dispersion close to 
the required ones (see Methods for details). Videos showing the imparted phases for the $n=0$ and $n=2$ metalenses are provided in the Supplementary Movie 1 and 2. The measured normalized focal length shifts and their theoretically predicted values from $\lambda$ $=470 \mathrm{~nm}$ to $670 \mathrm{~nm}$ are shown in Fig. 3(a). The latter was calculated by propagating the fields generated by the nanofins using Fresnel-Kirchhoff integration, neglecting the actual coupling between metalens elements. However, we also solved Maxwell's equations using FDTD simulations for a full metalens with $\mathrm{NA}=0.6$ and $6 \mu \mathrm{m}$ in diameter (due to the limitation of computational resources) to confirm achromatic focusing. Note that the effect of larger NA compensates for the smaller diameter. The results are shown in Supplementary Movie 3. Experimentally, the focal lengths at different wavelengths were obtained by measuring their intensity profiles (point spread functions) along the propagation direction (z-axis) of the incident beam in steps of 1 $\mu \mathrm{m}$, as shown in Fig. 3(b) to 3(d). The $z$-coordinate corresponding to the peak intensity value gives the focal length for a given wavelength.

We characterized the performance of these diffractive and achromatic metalenses in terms of their focal spot profiles (Fig. 3(e) and 3(f)). They were measured at the focal plane corresponding to an illumination wavelength of $470 \mathrm{~nm}$ (white dashed lines in Fig. 3(b) and 3(c)). The diffractive metalens shows significant defocusing when the wavelength is larger than $550 \mathrm{~nm}$ (Fig. 3(e)). In contrast, the focal spots of 
achromatic metalens at different wavelengths are diffraction-limited: their Strehl ratios are larger than 0.8 and the deviations of full-width at half-maximum (FWHM) are within $5 \%$ of the theoretical values (see Fig. S4). A real-time video showing the focal spot profiles as the incident wavelength is swept across the visible can be found in Supplementary Movie 4. It is important to note that although we only designed this achromatic metalens for a bandwidth of $120 \mathrm{~nm}$ centered at $530 \mathrm{~nm}$, due to the negligible focal length shift compared to its depth of focus $\left(\frac{\lambda}{N A^{2}}\right)$, the metalens maintains its focal spot profile for the entire visible spectrum from $470 \mathrm{~nm}$ to $670 \mathrm{~nm}$. This achromatic metalens can also focus incoherent white light source under a broadband halogen lamp illumination (Fig. S5).

We also fabricated achromatic and diffractive metalenses with larger diameters $(\mathrm{NA}=0.02$, diameter $=220 \mu \mathrm{m})$ and compared their imaging quality. The focal spot profiles of the achromatic metalens are shown in Fig. 4(a). A real-time video of focal spot profile measurement is provided in Supplementary Movie 5. Figure 4(b) shows the images of a standard USAF resolution target obtained from the achromatic metalens, under various laser illumination wavelengths with a bandwidth of $40 \mathrm{~nm}$, corresponding to the bandwidth of a typical LED light source (see Methods for experimental setup). Control experiments using the diffractive metalens are in Fig. S6. The test target was fixed at the focal plane corresponding to illumination at $\lambda=470 \mathrm{~nm}$. In Fig. 4(b), a 
slight decrease of contrast in the images at red wavelengths is observed since the feature size of the target $(\sim 15 \mu \mathrm{m})$ is close to the diffraction limit of the achromatic metalens; there is also a decrease in efficiency of the metalens at red wavelengths.

Additionally, we demonstrate white light imaging using a broadband illumination source (white light laser) from 470-670 nm. The images of the USAF target and Siemens star are shown in Fig. 4(c) and 4(d). These images show that chromatic aberration is well-corrected even under white light illumination, and that the metalens is able to achieve high imaging quality over a few square millimeters, corresponding to a 30 degrees field of view. Note that the patterns at the center of the USAF target and the Siemens star have feature sizes smaller than the resolution of the achromatic metalens. We measured the focal spots of the metalens for different angles of incidence, and found that their Strehl ratios are larger than 0.8 till an incident angle of about 15 degrees (Fig. S7). The color of these white light images has a bluish tinge because of the wavelength-dependent efficiency of the metalens (see Methods for efficiency measurement). We measured about $20 \%$ efficiency around $500 \mathrm{~nm}$, against a theoretically predicted value of 50\% (Fig. S8(a)). This deviation likely results from fabrication errors and the coupling between metalens elements. We simulate the phase of each element (coupled waveguides) using periodic boundary conditions. This approximation ignores near-field coupling to adjacent metalens elements, thereby 
introducing a perturbation on the wavefront and an attendant reduction in efficiency.

This effect can be taken into account with comprehensive full lens simulation followed by optimization to increase efficiency ${ }^{35,36}$. Note that the metalens efficiency is lower compared to our prior work ${ }^{37}$, because in order to cover a larger range of group delay, some low polarization conversion efficiency elements must be chosen (see Fig. S3). The metalens efficiency can be increased by introducing more complicated nanostructures to increase the freedom of design parameters, or by choosing highly efficient elements at the expense of reducing metalens diameter. We fabricated and measured two metalenses with smaller diameters with efficiencies of about $40 \%$ (Fig. $\mathrm{S} 8(\mathrm{~b}))$.

Our design principle can be applied to other regions of the electromagnetic spectrum ${ }^{38,39}$. In addition, as mentioned previously, realizing achromatic metalenses with larger diameters and higher numerical apertures requires a larger range of group delay supported by various combinations of nanofins with different dimensions. This can be realized, as shown in Eq. 5, by different dispersion engineering approaches ${ }^{40-43}$ or by simply increasing the height of the nanofins ${ }^{44-47}$. The authors of Ref. 47 have demonstrated $\mathrm{TiO}_{2}$ nanostructures with $\sim 4.5 \mu \mathrm{m}$ height corresponding to a group delay of about 37 femto-seconds. Cascading layers of metalenses can further increase the total group delay; moreover, this introduces an additional degree of freedom to correct for 
monochromatic aberrations (coma, field curvature, astigmatism etc.) within a large field of view ${ }^{36,48,49}$. Finally, one can combine a metalens, which acts as an aberration corrector with a refractive spherical lens. This is particularly promising: one would be able to simultaneously correct chromatic and monochromatic aberrations of the spherical lens, while maintaining the benefits of a large lens aperture and a small chromatic focal length shift. An example is given in Fig. S9 for a commercial low-cost spherical lens (LA4966, Thorlabs Inc.) with a diameter of $5.4 \mathrm{~mm}$ and NA $=0.1$ attached to a dispersion-engineered metalens. Our calculations show that the focal length shift of the refractive/metalens doublet is similar to that of triplet lenses if one incorporates group delay dispersion engineering in the design of the metalens. Moreover, the metalens enables the spherical lens to achieve diffraction-limited achromatic focusing across the visible spectrum from $450 \mathrm{~nm}$ to $700 \mathrm{~nm}$, a feat which traditionally requires cascading of many refractive lenses of various glass materials ${ }^{50}$. The required group delay for such metalens is about 22 femto-seconds, only four times larger than what we can realize with 600-nm-height nanofins.

\section{Conclusions}

By simultaneously controlling the phase, group delay and group delay dispersion, we have demonstrated dispersion-tailored metalenses in transmission over a large continuous bandwidth in the visible region. This represents a significant advance in the 
state-of-the-art for metalenses, which have traditionally been limited in their applications due to bandwidth, particularly in the visible. We have demonstrated an achromatic planar meta-lens $(\mathrm{NA}=0.2)$ capable of focusing light to the diffractionlimit from 470 to $670 \mathrm{~nm}$, and an achromatic meta-lens $(\mathrm{NA}=0.02)$ for imaging. We have also shown that a metalens with tailored dispersion can transform a low-cost spherical lens into a diffraction-limited, achromatic lens across the entire visible. These achromatic and dispersion-tailored metalenses can find numerous applications across industry and scientific research, such as in lithography, microscopy, endoscopy and virtual/augmented reality. 


\section{Methods}

Design and simulation. Simulations were performed using commercial package from Lumerical Inc. (FDTD and Eigenmode solutions). A large number of elements, each comprising of one or two nanofins of different lengths, widths and arrangements, were simulated under circularly polarized illumination to build a library. Periodic and perfectly matched layer boundary conditions were used along the transverse and longitudinal directions with respect to the propagation of light. For each simulation, the far-field electric field of an element was recorded, then normalized to the field with substrate only. The polarization conversion efficiency and phase spectrum were obtained from the normalized electric field.

To design the achromatic meta-lenses, we linearly fit the phase spectrum of each element in our library at the design wavelength $(530 \mathrm{~nm})$ within a $120 \mathrm{~nm}$ bandwidth to obtain the group delay. Any element that has an R-squared value and polarization conversion efficiency less than 0.99 and 5\% was dropped. The linear fitting with high R-squared value ensures that all selected elements from the library fulfill the requirement for realizing achromatic meta-surfaces with larger than $120 \mathrm{~nm}$ bandwidth. Our simulations and measurements of the point spread function have shown that the metalens remains achromatic with up to a bandwidth of $200 \mathrm{~nm}$. Finally, for each coordinate of the achromatic metalens, the required group delay was achieved by 
positioning the corresponding suitably rotated element in order to satisfy the required phase profile at wavelength $\lambda=530 \mathrm{~nm}$. For the metalens with $\mathrm{n}=2$, we fit the phase spectrum with a quadratic polynomial to obtain group delay and group delay dispersion. Two different offset values are chosen by the particle swarm algorithm to best satisfy the required relative group delay and group delay dispersion (see Fig. S10 for details). Relation between required group delay and metalens radius and NA. The required range of group delay for an achromatic metalens is given by

$$
\left|\frac{\partial \varphi}{\partial \omega}\right|=\frac{R \cdot N A}{2 c}
$$

where $c$ is light speed and $R$ is the metalens radius, as obtained by substituting numerical aperture $N A=R / F \quad$ (small-angle approximation) into Eq. 1 after differentiating. The range of group delays given by all elements is related to the diameter and numerical aperture of the achromatic metalens.

Measurement. The meta-lenses were characterized using a custom-built microscope mainly consisting of a tunable laser (SuperK and Varia from NKT) and an Olympus objective lens $(100 \times, \mathrm{NA}=0.95)$ paired with a tube lens (focal length $f=180 \mathrm{~mm})$ to form an image on a camera. The objective lens was moved 1 um per step by a motorized stage (Märzhäuser, MA42) controlled by Labview to record the intensity distributions along the beam propagation direction on a monochromatic camera (Thorlabs, DCC1545M) for different wavelengths. Subsequently, these images are stacked to 
obtain Fig. 3(b) to 3(d). For measuring the focal spot profiles shown in Fig. 3(e) and 3(f), the camera was replaced by another with smaller pixel size (Edmund, EO-5012). For all measurements, a pair of crossed circular polarizers were used to reduce background noise. A schematic of the experimental setup and details for Fig. 4 can be found in Fig. S11.

To measure metalens efficiency, we measured the power in the focal spot (using an iris aperture to block background light at the imaging plane) and divided it by the power of circularly polarized incident light passing through an aperture with the same diameter as the metalens.

\section{Data availability}

The libraries that support the design of metalenses are available from the corresponding author on reasonable request.

\section{Additional Information}

Supplementary information is available in the online version of the paper. Reprints and permissions information is available online at www.nature.com/reprints. Correspondence and requests for materials should be addressed to W. T. C. and F. C.

\section{Supplementary Materials:}

Figure S1-S11

Movies S1-S5

\section{Author contributions:}


W. T. C. and F.C. conceived of the study. A. Y. Z. fabricated the metalenses. W. T. C., V. S., M. K., Z. J. and E. L. performed simulations and developed codes. W. T. C., A. Y. Z. and E. L. measured the metalenses. W. T. C., A. Y. Z., M. K. and F. C. wrote the manuscript. All authors discussed the results and commented on the manuscript.

\section{Competing financial interests}

The authors declare no competing financial interests

\section{Acknowledgements}

This work was supported by the Air Force Office of Scientific Research (MURI, grant\# FA9550-14-1-0389 and grant\# FA9550-16-1-0156). This work was performed in part at the Center for Nanoscale Systems (CNS), a member of the National Nanotechnology Coordinated Infrastructure (NNCI), which is supported by the National Science Foundation under NSF award no. 1541959. CNS is part of Harvard University. Federico Capasso gratefully acknowledges a gift from Huawei Inc. under its HIRP FLAGSHIP program. We thank Yao-Wei Huang and Jared Sisler for their helps in measurement and in simulations, respectively. 


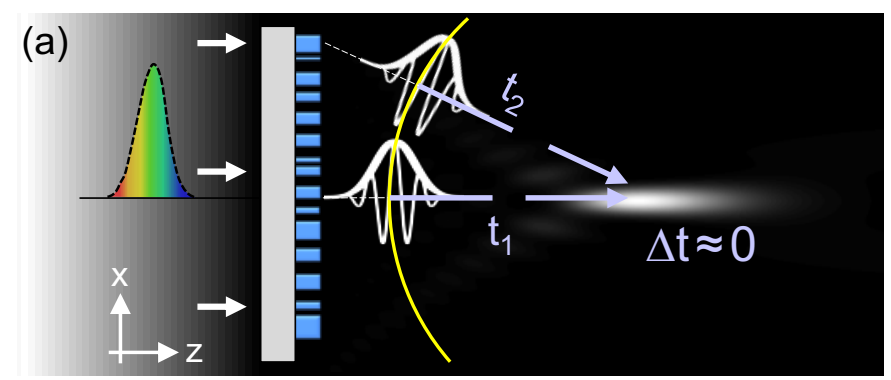

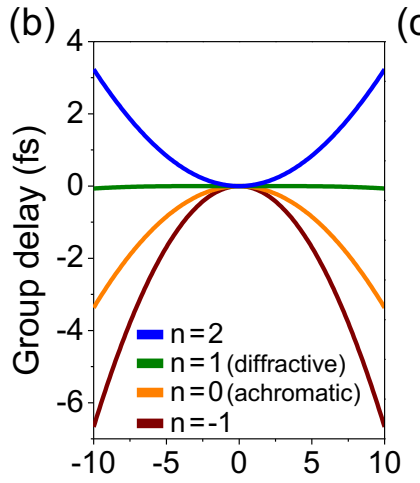

Radial coordinate $(\mathrm{m})$ (c)

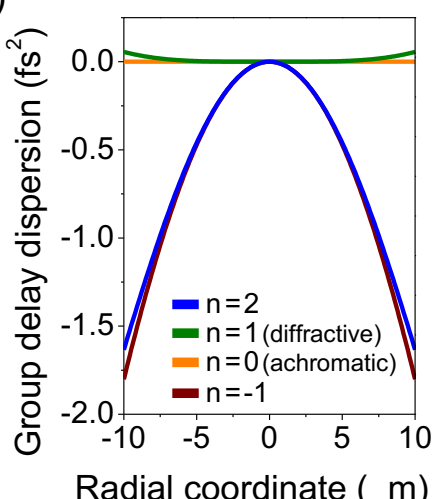

Figure 1: Dispersion engineering of achromatic and chromatic metalenses. (a)

Schematic of an achromatic metalens. To realize achromatic focusing, the phase profile of $\varphi(r, \omega)$ must satisfy Eq. 1 in the text. The metalens is designed to provide spatially dependent group delays such that wavepackets from different locations arrive simultaneously at the focus. The yellow line shows the spherical wavefront. (b) Required relative group delays as a function of metalens coordinate. The focal length is parametrized as $F(\omega)=k \omega^{n}$. Note that the NA is a function of wavelength for $n \neq 0$ due to the change in focal length. Depending on the value of $\mathrm{n}$, the metalens can be designed as achromatic $(\mathrm{n}=0)$ or chromatic with focal length inversely proportional to wavelength ( $\mathrm{n}=1$, dispersion similar to Fresnel lenses), or proportional to wavelength $(n=-1)$. The case of $n=2$ exhibits stronger dispersion. These metalenses 
have a diameter of $20 \mu \mathrm{m}$ and a focal length of $49 \mu \mathrm{m}$ at $\lambda=530 \mathrm{~nm}$. (c) Required relative group delay dispersion of the same metalenses. 
(a)
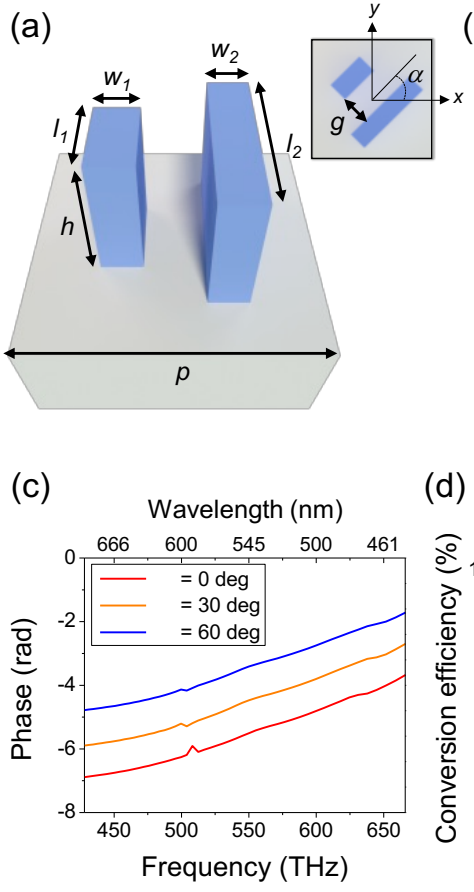

(b)

(d)
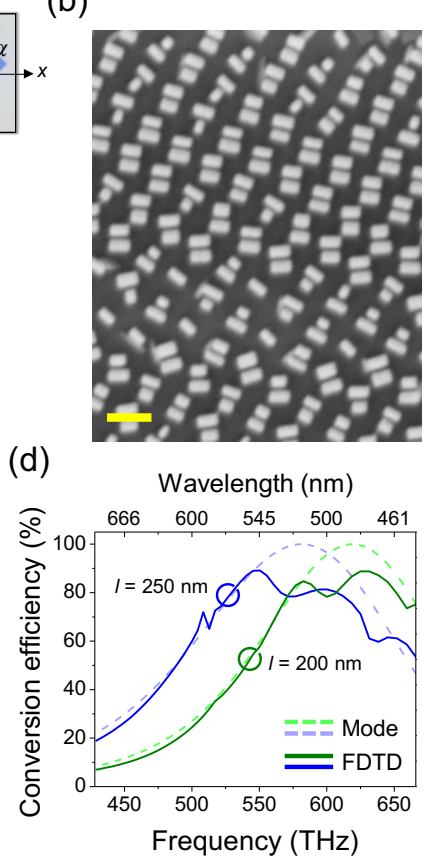

(e)

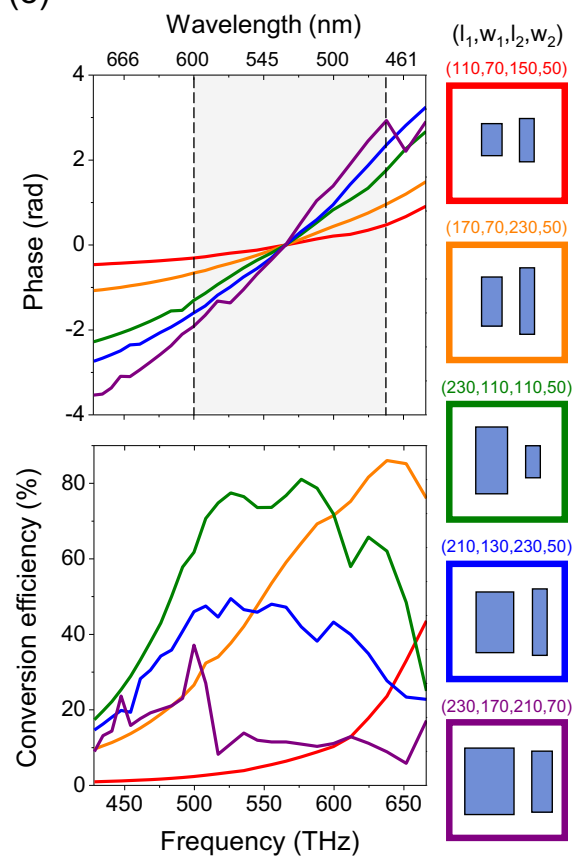

Figure 2: Optical properties of nanofins and scanning electron micrograph. (a)

Schematic of a metalens element. The element consists of one or more $\mathrm{TiO}_{2}$ nanofins of varying dimensions but equal height $h=600 \mathrm{~nm}$, evenly spaced by a distance $p=$ $400 \mathrm{~nm}$. The gap between nanofins is $g=60 \mathrm{~nm}$. The length $l$, width $w$, height $h$ and rotation angle $\alpha$ are also shown; the subscripts denote the left and right nanofin, respectively. The nanofins are rotated with respect to the center of the square $(400 \times$ $400 \mathrm{~nm}^{2}$ ). (b) Scanning electron micrograph of a region of a fabricated metalens. Scale bar: $500 \mathrm{~nm}$. For more images of the metalens, see Fig. S2. (c) and (d) Simulation results for a single nanofin. (c) Phase plots as a function of frequency for different rotation angles for nanofins with $l=250 \mathrm{~nm}$ and $w=80 \mathrm{~nm}$. (d) A comparison of polarization conversion efficiency for different nanofin lengths from FDTD 
calculations (solid lines) versus Mode Solutions (dashed lines). The lengths of the nanofins are labeled; they have a constant width $w=80 \mathrm{~nm}$. (e) Phase spectra and polarization conversion efficiencies for five different elements showing the tunability of the group delay by changing the lengths and widths of nanofins. The shaded region marks the design bandwidth of $120 \mathrm{~nm}$. Each colored curve corresponds to its element schematically shown on the right. The parameters $\left(l_{1}, w_{1}, l_{2}, w_{2}\right)$ of each nanofins are labelled; all dimensions are in nanometers. The elements in the colored squares are located at different radial positions from the edge of the metalens (red square) to the center (purple square), such that the corresponding group delay (slop of the phase versus angular frequency plot) increases from the edge to the center. This ensures achromatic focusing as illustrated in Fig. 1(a). 

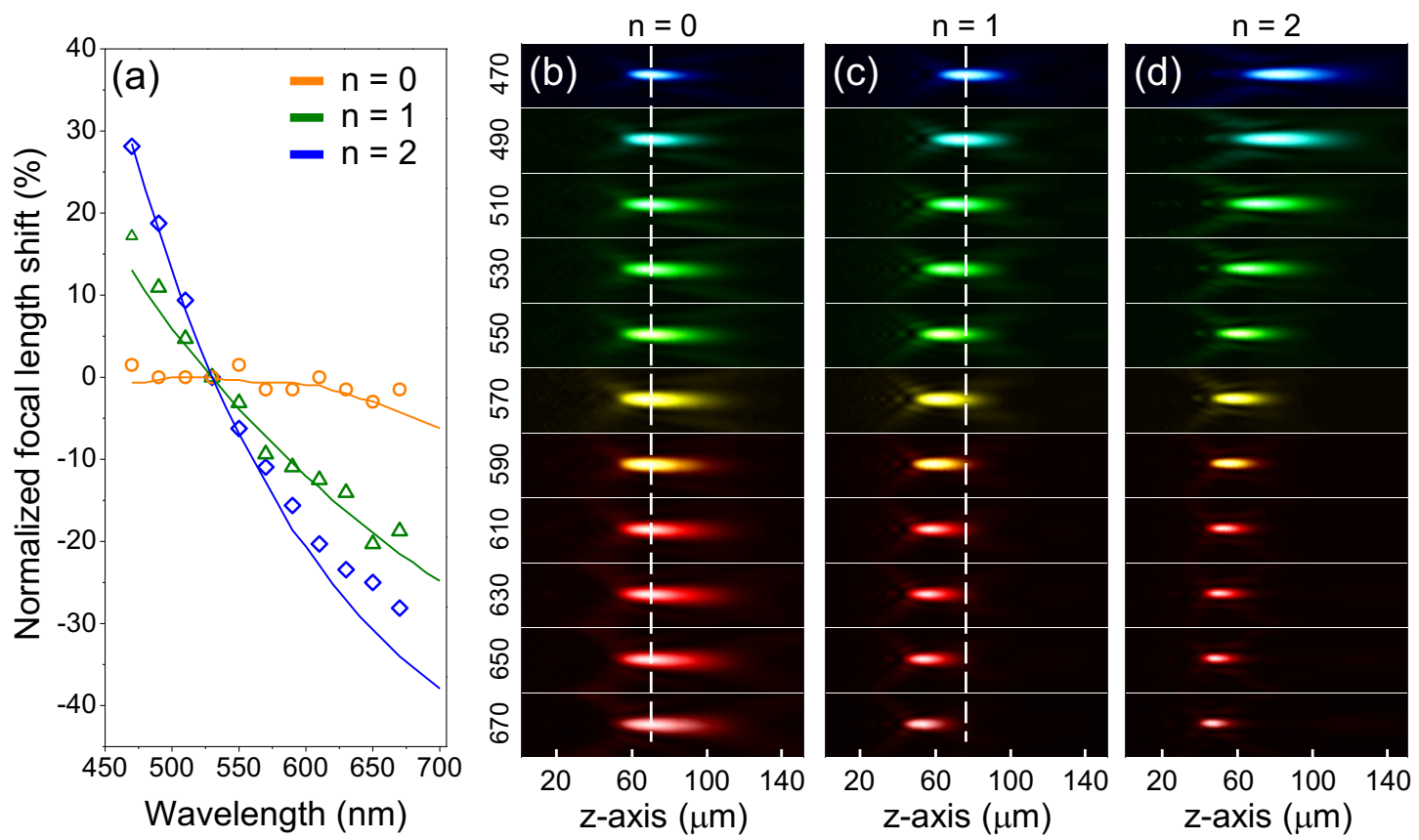

(e)
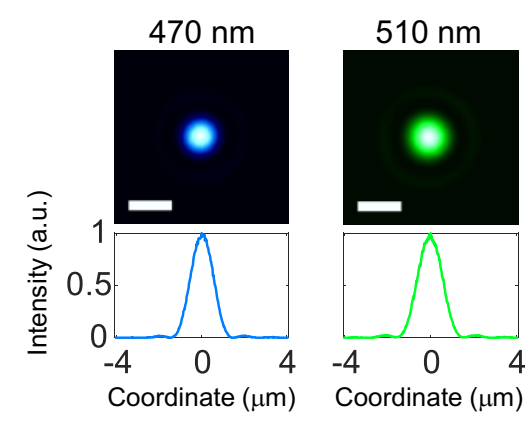

Diffractive Metalens

(f)
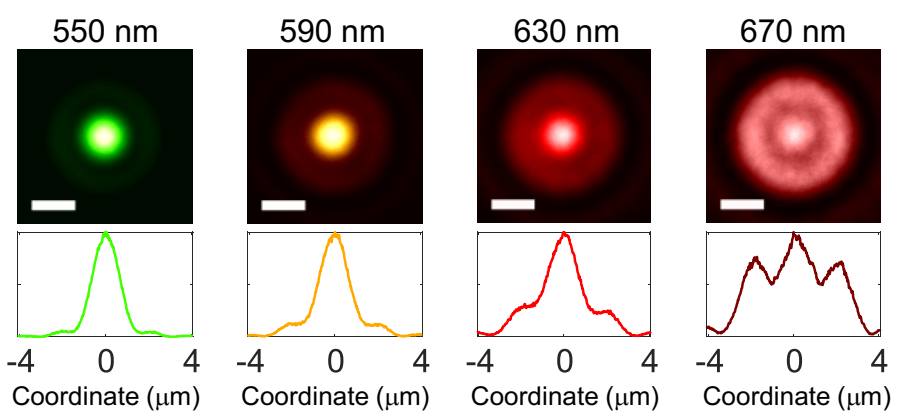

\section{Achromatic Metalens}
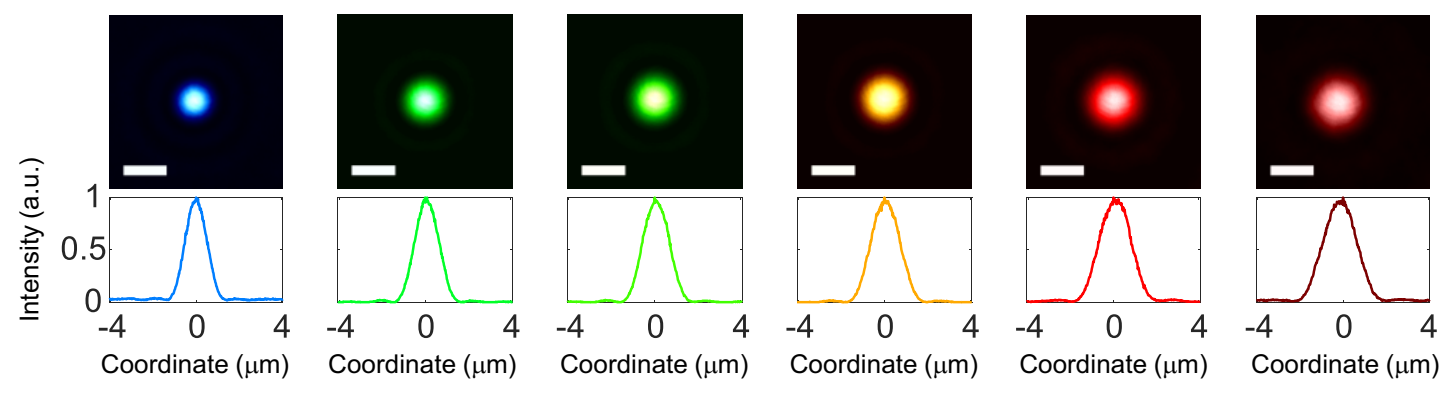

Figure 3: Measured focal length shifts and intensity distributions of metalenses.

(a) Experimentally measured normalized focal length shifts (symbols) compared to simulations (lines) for metalenses with different values of $n$. Incident light was collimated and at normal incidence. The metalenses were designed at wavelength $\lambda=$ 
$530 \mathrm{~nm}$ with NA $=0.2$ and focal length of $63 \mu \mathrm{m}$. (b) to (d) Measured intensity distributions in linear scale (in false colors corresponding to their respective wavelengths) in the $x-z$ plane. (b), (c) and (d) correspond to metalenses with $n=0$ (achromatic), 1 and 2, respectively. The wavelengths of incidence are denoted on the left. The direction of incidence is towards the positive $z$-axis. (e) and (f) Normalized intensity profiles along the white dashed lines of (c) and (b) for diffractive and achromatic metalenses, respectively. The white dashed lines pass through the center of focal spots in case of $\lambda=470 \mathrm{~nm}$. Scale bar: $2 \mu \mathrm{m}$. 

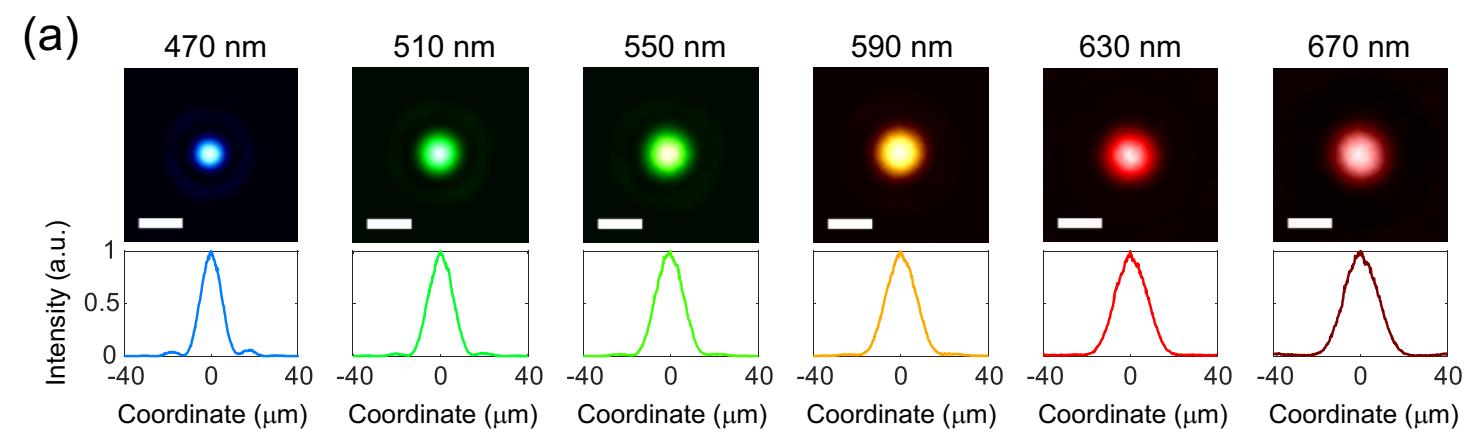

(b)
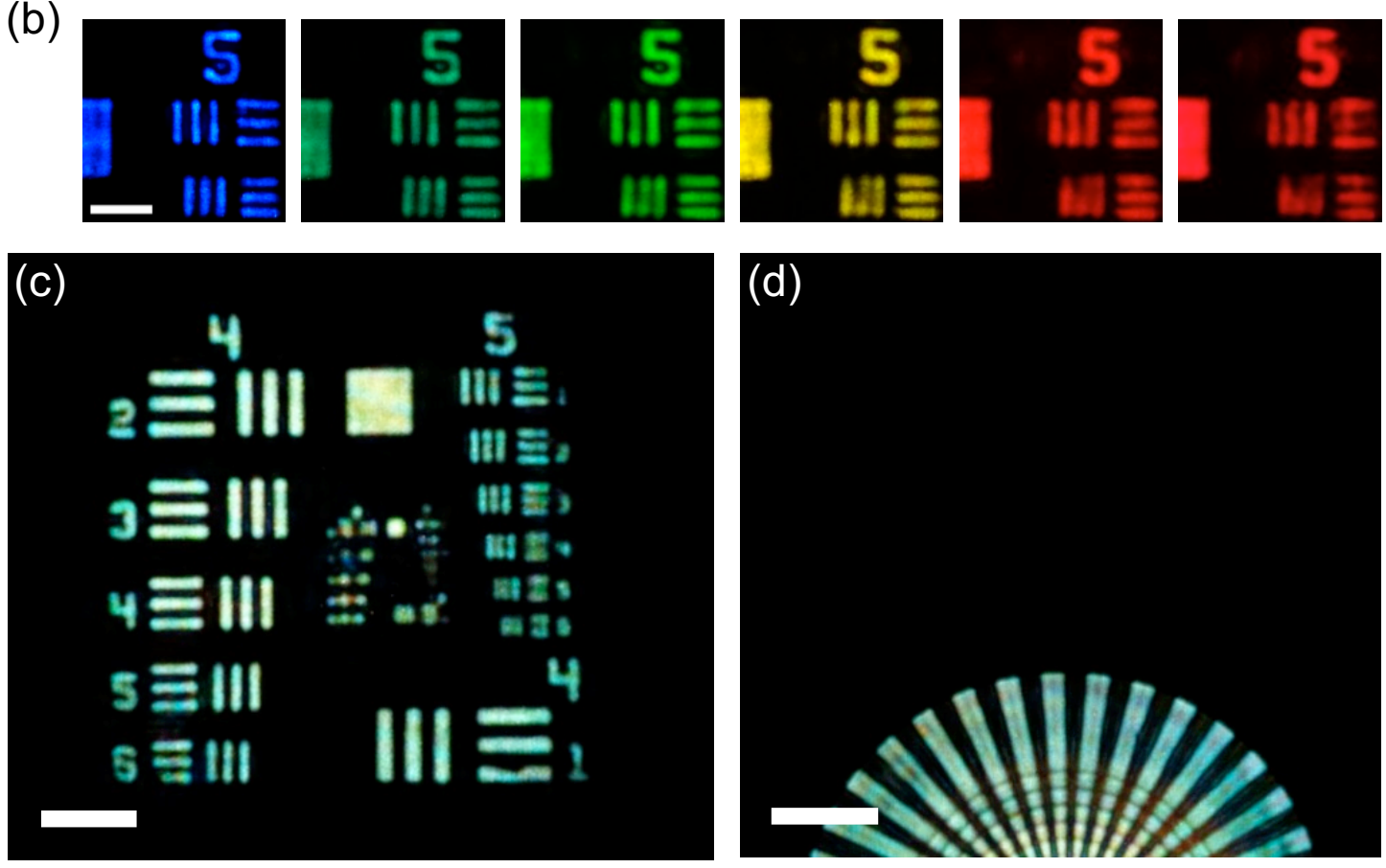

Figure 4: Focal spot profiles and imaging using an achromatic metalens under

different illumination wavelengths. The achromatic metalens has a diameter of 220

$\mu \mathrm{m}$ and NA of 0.02 . The light source is a supercontinuum laser with tunable center wavelength and bandwidth. (a) Experimentally measured focal spot profiles. The illumination wavelengths with about $5 \mathrm{~nm}$ bandwidth are denoted on the top. Scale bar: $20 \mu \mathrm{m}$. (b) Images of 1951 United States Air Force resolution target formed by the achromatic metalens. The resolution target (Thorlabs, R1L1S1N) was fixed at the focal 
plane corresponding to an incident wavelength $\lambda=470 \mathrm{~nm}$. The line widths of upper

and lower bars are 15.6 and $14 \mu \mathrm{m}$, respectively. Scale bar: $100 \mu \mathrm{m}$. (c) Images of the same Air Force target using the achromatic metalens under an illumination bandwidth of $200 \mathrm{~nm}$ centered at $570 \mathrm{~nm}$. (d) The image of the Siemens star pattern under the same illumination condition in (c). The outer radius is $500 \mu \mathrm{m}$. The metalens provides about $50 \times$ magnification. Scale bars are $200 \mu \mathrm{m}$. Figure S6 shows that a chromatic metalens of same diameter and NA generates significantly blurred images, in contrast to the achromatic lens.

\section{References:}

1 Chen, W. T. et al. Immersion Meta-Lenses at Visible Wavelengths for Nanoscale Imaging. Nano Lett. 17, 3188-3194 (2017).

2 Khorasaninejad, M. et al. Multispectral Chiral Imaging with a Meta-lens. Nano Lett. 16, 45954600 (2016).

3 Khorasaninejad, M. et al. Metalenses at visible wavelengths: Diffraction-limited focusing and subwavelength resolution imaging. Science 352, 1190-1194 (2016).

4 Schonbrun, E., Seo, K. \& Crozier, K. B. Reconfigurable Imaging Systems Using Elliptical Nanowires. Nano Lett. 11, 4299-4303 (2011).

5 Chen, W. T. et al. Integrated plasmonic metasurfaces for spectropolarimetry. Nanotechnology 27, 224002 (2016).

6 Balthasar Mueller, J. P., Leosson, K. \& Capasso, F. Ultracompact metasurface in-line polarimeter. Optica 3, 42-47 (2016).

7 Pors, A., Nielsen, M. G. \& Bozhevolnyi, S. I. Plasmonic metagratings for simultaneous determination of Stokes parameters. Optica 2, 716-723 (2015).

8 Chen, W. T. et al. Generation of wavelength-independent subwavelength Bessel beams using metasurfaces. Light Sci. Appl. 6, e16259 (2017).

9 Lin, D., Fan, P., Hasman, E. \& Brongersma, M. L. Dielectric gradient metasurface optical elements. Science 345, 298-302 (2014).

$10 \mathrm{Wu}$, P. C. et al. Versatile Polarization Generation with an Aluminum Plasmonic Metasurface. Nano Lett. 17, 445-452 (2017). 
Zhao, Z. et al. Multispectral optical metasurfaces enabled by achromatic phase transition. Sci. Rep. 5, 15781 (2015).

$\mathrm{Pu}, \mathrm{M}$. et al. Catenary optics for achromatic generation of perfect optical angular momentum. Sci. Adv 1, e1500396 (2015).

Ye, W. et al. Spin and wavelength multiplexed nonlinear metasurface holography. Nat. Commun. 7, 11930 (2016).

Zheng, G. et al. Metasurface holograms reaching 80\% efficiency. Nat. Nanotechnol. 10, 308312 (2015). (2016).

Aieta, F., Kats, M. A., Genevet, P. \& Capasso, F. Multiwavelength achromatic metasurfaces by dispersive phase compensation. Science 347, 1342-1345 (2015). Hu, J., Liu, C.-H., Ren, X., Lauhon, L. J. \& Odom, T. W. Plasmonic Lattice Lenses for Multiwavelength Achromatic Focusing. ACS nano 10, 10275-10282 (2016). Avayu, O., Almeida, E., Prior, Y. \& Ellenbogen, T. Composite functional metasurfaces for multispectral achromatic optics. Nat. Commun. 8, 14992 (2017).

Faklis, D. \& Morris, G. M. Spectral properties of multiorder diffractive lenses. Appl. Opt. 34, 2462-2468 (1995).

Li, Y. et al. Achromatic flat optical components via compensation between structure and material dispersions. Sci. Rep. 6, 19885 (2016).

Li, M., Al-Joumayly, M. A. \& Behdad, N. Broadband true-time-delay microwave lenses based on miniaturized element frequency selective surfaces. IEEE Trans. Antennas Propag. 61, 1166-1179 (2013).

Cheng, J. \& Mosallaei, H. Truly achromatic optical metasurfaces: a filter circuit theory-based design. J. Opt. Soc. Am. B 32, 2115-2121 (2015). Ajioka, J. S. Broadband group delay waveguide lens. USA patent 126075 (1982). Khorasaninejad, M. et al. Achromatic Metalens over $60 \mathrm{~nm}$ Bandwidth in the Visible and Metalens with Reverse Chromatic Dispersion. Nano Lett. 17, 1819-1824 (2017). Shrestha, S., Overvig, A. \& Yu, N. Broadband Achromatic Metasurface Lenses. Conference on Lasers and Electro-Optics (CLEO), FM1H.3,San Jose, California (2017).

Arbabi, E., Arbabi, A., Kamali, S. M., Horie, Y.\& Faraon, A. Controlling the sign of chromatic dispersion in diffractive optics with dielectric metasurfaces. Optica 4, 625-632 (2017). Wang, S. et al. Broadband achromatic optical metasurface devices. Nat. Commun. 8 (2017). Chen, L., Shakya, J. \& Lipson, M. Subwavelength confinement in an integrated metal slot waveguide on silicon. Opt. Lett. 31, 2133-2135 (2006).

Zhang, L. et al. Silicon waveguide with four zero-dispersion wavelengths and its application in on-chip octave-spanning supercontinuum generation. Opt. Express 20, 1685-1690 (2012). 
Devlin, R. C., Khorasaninejad, M., Chen, W. T., Oh, J. \& Capasso, F. Broadband highefficiency dielectric metasurfaces for the visible spectrum. Proc. Natl. Acad. Sci. U.S.A. 113, 10473-10478 (2016).

Yu, N. \& Capasso, F. Flat optics with designer metasurfaces. Nat. Mater. 13, 139-150 (2014). Karagodsky, V., Sedgwick, F. G. \& Chang-Hasnain, C. J. Theoretical analysis of subwavelength high contrast grating reflectors. Opt. Express 18, 16973-16988 (2010). Wang, S. \& Magnusson, R. Theory and applications of guided-mode resonance filters. Appl. Opt. 32, 2606-2613 (1993).

Escuti, M. J., Kim, J. \& Kudenov, M. W. Controlling light with geometric-phase holograms. Optics and Photonics News 27, 22-29 (2016).

Byrnes, S. J., Lenef, A., Aieta, F. \& Capasso, F. Designing large, high-efficiency, highnumerical-aperture, transmissive meta-lenses for visible light. Opt. Express 24, 5110-5124 (2016).

Lin, Z., Groever, B., Capasso, F., Rodriguez, A. W. \& Lončar, M. Topology Optimized Multilayered Meta-optics. Preprint at https://arxiv.org/abs/1706.06715 (2017).

Khorasaninejad, M. et al. Polarization-insensitive metalenses at visible wavelengths. Nano Lett. 16, 7229-7234 (2016).

Wang, S., Lai, J., Wu, T., Chen, C. \& Sun, J. Wide-band achromatic flat focusing lens based on all-dielectric subwavelength metasurface. Opt. Express 25, 7121-7130 (2017). Yang, J. et al. Broadband planar achromatic anomalous reflector based on dispersion engineering of spoof surface plasmon polariton. Appl. Phys. Lett. 109, 211901 (2016).

Dastmalchi, B., Tassin, P., Koschny, T. \& Soukoulis, C. M. Strong group-velocity dispersion compensation with phase-engineered sheet metamaterials. Physical Review B 89, 115123 (2014).

1 Decker, M. et al. High-efficiency dielectric Huygens' surfaces. Adv. Opt. Mater 3, 813-820 (2015).

Caloz, C. Metamaterial dispersion engineering concepts and applications. Proc. IEEE 99, 1711-1719 (2011).

3 Qu, C. et al. Tailor the functionalities of metasurfaces based on a complete phase diagram. Phys. Rev. Lett. 115, 235503 (2015).

Gorelick, S., Guzenko, V. A., Vila-Comamala, J. \& David, C. Direct e-beam writing of dense and high aspect ratio nanostructures in thick layers of PMMA for electroplating.

Nanotechnology 21, 295303 (2010).

5 Lee, S., Park, B., Kim, J. S. \& Kim, T.-i. Designs and processes toward high-aspect-ratio nanostructures at the deep nanoscale: unconventional nanolithography and its applications. Nanotechnology 27, 474001 (2016).

Gissibl, T., Thiele, S., Herkommer, A. \& Giessen, H. Two-photon direct laser writing of ultracompact multi-lens objectives. Nat. Photonics 10, 554-560 (2016). 
47 Shkondin, E. et al. Fabrication of high aspect ratio $\mathrm{TiO} 2$ and $\mathrm{A} 12 \mathrm{O} 3$ nanogratings by atomic layer deposition. J. Vac. Sci. Technol 34, 031605 (2016).

48 Groever, B., Chen, W. T. \& Capasso, F. Meta-Lens Doublet in the Visible Region. Nano Lett. 17, 4902-4907 (2017).

49 Arbabi, A. et al. Miniature optical planar camera based on a wide-angle metasurface doublet corrected for monochromatic aberrations. Nat. Commun. 7, 13682 (2016).

50 Herzberger, M. \& McClure, N. R. The design of superachromatic lenses. Appl. Opt. 2, 553560 (1963). 